over the sky. The Hubble law has been confirmed with ever-improving precision.

Today, Arp is left bitter and combative, with only a handful of astronomers such as Fred Hoyle and Geoff Burbidge taking him seriously. In this book he collects together all the best examples of his wondrous phenomena and an unimpressive and unconvincing assembly it makes. The 'lines' and 'concentrations' of quasars appear no more unusual than would be expected by chance, given that Arp has worked extremely hard to find these examples. The high-redshift galaxies near foreground objects always look just like more distant, background objects. Every so often Arp stumbles on some real phenomenon, such as the interactions of his 1966 Atlas or the optical jet of M87, but even then his explanation of it always has to be totally bizarre.

At one point Arp resorts to a rather sad piece of sophistry. Discussing the galaxies of Stephan's Quintet he remarks that a supernova had been discovered in 1971 in NGC 7319, the galaxy in the group which has an anomalously high redshift. Rather than accept that the faintness of the supernova supported a higher distance for the high-redshift galaxy, Arp concludes that: "stars in the anomalously redshifted systems simply do not get as bright as in galaxies of lower intrinsic redshift". This is sad because in 1961 Arp was the first to apply the Baade-Wesselink expansion parallax method to supernovae, and so could be called the father of the supernova distance method.

Arp claims that he has been harshly treated and kept off telescopes and out of astronomical journals. Yet he has managed to publish over 40 papers on his exotica over the past 20 years. He was president of the Astronomical Society of the Pacific from 1980 to 1983 , and has received a sprinkling of prizes and awards. This book illustrates the maxim that an obsession can ruin your whole life.

Michael Rowan-Robinson is Professor of Astrophysics in the Astronomy Unit, Oueen Mary College, Mile End Road, London E1 $4 N S, U K$

\section{The universal rover}

\section{Owen Gingerich}

The World Within the World. By John D. Barrow. Clarendon: 1988. Pp. 398. £20, $\$ 24.95$.

How does the uniformity of nature depend on the quantization of energy levels? I can only admire a 'popularization' that has the ingenuity to ask such a question. Here John Barrow, an astronomer at the University of Sussex, not only asks the question, but provides a notably clear answer: if the electrons orbiting protons could have any arbitrary speeds, then each hydrogen atom would be different, and "the uniformity of Nature an unrealized idealization".

In this wonderfully wide-ranging excursion, Barrow has brought a freshness of descriptive metaphor to some of the more arcane topics of modern physical science such as the Einstein-Podolsky-Rosen paradox, gauge theories, chaos, superstrings and the inflationary universe. Barrow explores not only a wide gamut of topics in the zone between physics and astronomy, but he wrestles with their philosophical contexts as well. His "world within the world" is the submicroscopic quantum framework within the largescale cosmos, so strangely incompatible with the relativistic picture of the vast astronomical Universe and yet so essential to its understanding. As he describes the march of science, he circles around a far more difficult question: are there really laws of nature out there to be discovered, or are we just imposing our own human
History of Time. Hawking applies a coordinate transformation to the singularity of the Big Bang, expands the time coordinate back to infinity, and glibly announces an originless Universe, asking "What place, then, for a creator?". Barrow, in contrast, discusses the question of singularities at some length, and concludes that until we better understand the nature of time, we will really not know how to answer those who ask whether the Universe is of finite or infinite age.

Although it achieves a sophisticated level of popularization, Barrow's World is nevertheless an uneven book. The conversation between a mathematical intuitionalist and a platonist is a confusing detour, and Barrow's road map of philosophical routes is too terse to set his readers onto clear paths. In his assessment of Thomas Kuhn's paradigmatic structure of scientific explanations, and elsewhere, he subtly suggests that the highest philosophical calling of a physicist is to be a realist. Yet his sympathetic discussion of the 'many worlds' interpretation of quantum mechanics, his description of multiple dimensions of the Universe, and his emphasis on metaphysical and aesthetic criteria as guides to theoretical speculation seem to make him far more the idealist.

Indeed, after Barrow's rewarding journey to the limits of space and time, his conclusion is not all that unexpected:

[these are] extraordinary problems, and they possess extraordinary solutions which will require extraordinary methods to coax from the Universe. If our methods ultimately fail, then any boundary between fundamental science and metaphysical theology will become increasingly difficult to draw. Sight must give way to faith. Confronted with an emotionally satisfying mathematical scheme which is 'simple' enough to command universal assent, but esoteric enough to admit no means of experimental test then, closeted within our world within the world, we might simply have to believe it.

Owen Gingerich is a Professor in the HarvardSmithsonian Center for Astrophysics, 60 Garden Street, Cambridge, Massachusetts 02138 , USA the sleight of hand that characterizes Stephen Hawking's best-selling A Brief

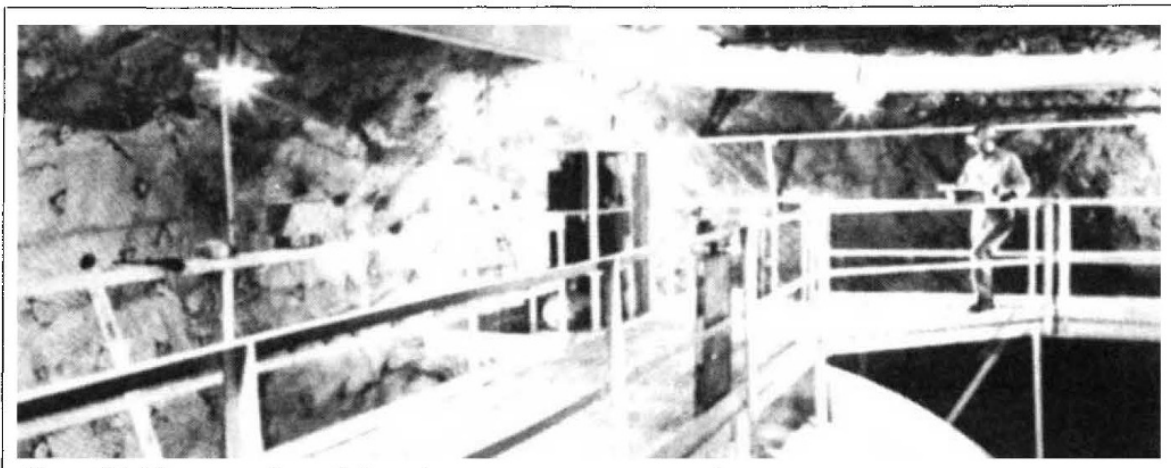

Deep thinking - a view of the solar neutrino experiment located at a depth of 4,850 feet in the Homestake gold mine. The picture is reproduced from Particle Physics: A Los Alamos Primer edited by N.C. Cooper and G.B. West, published earlier this year by Cambridge University Press. Price is hbk £30,\$49.50; pbk £9.95, \$14.95. 